\title{
Contrast-distorted image quality assessment based on curvelet domain features
}

\author{
Ismail Taha Ahmed ${ }^{1}$, Chen Soong Der ${ }^{2}$, Baraa Tareq Hammad ${ }^{3}$, Norziana Jamil ${ }^{4}$ \\ ${ }^{1,3}$ College of Computer Sciences and Information Technology, University of Anbar, Anbar, Iraq \\ ${ }^{2}$ College of Graduate Studies, Universiti Tenaga Nasional, Malaysia \\ ${ }^{4}$ College of Computing and Informatics, Universiti Tenaga Nasional, Malaysia
}

\begin{tabular}{l}
\hline Article Info \\
\hline Article history: \\
Received Sep 11, 2019 \\
Revised Jul 26, 2020 \\
Accepted Sep 24, 2020 \\
\hline
\end{tabular}

\section{Keywords:}

Contrast-distorted image

IQAs

NR-IQA

NR-IQA-CDI

NR-IQA-CDI-CvT

\begin{abstract}
Contrast is one of the most popular forms of distortion. Recently, the existing image quality assessment algorithms (IQAs) works focusing on distorted images by compression, noise and blurring. Reduced-reference image quality metric for contrast-changed images (RIQMC) and no reference-image quality assessment (NR-IQA) for contrast-distorted images (NR-IQA-CDI) have been created for CDI. NR-IQA-CDI showed poor performance in two out of three image databases, where the Pearson correlation coefficient (PLCC) were only 0.5739 and 0.7623 in TID2013 and CSIQ database, respectively. Spatial domain features are the basis of NR-IQA-CDI architecture. Therefore, in this paper, the spatial domain features are complementary with curvelet domain features, in order to take advantage of the potent properties of the curvelet in extracting information from images such as multiscale and multidirectional. The experimental outcome rely on K-fold cross validation (K ranged 2-10) and statistical test showed that the performance of NR-IQACDI rely on curvelet domain features (NR-IQA-CDI-CvT) significantly surpasses those which are rely on five spatial domain features.
\end{abstract}

This is an open access article under the CC BY-SA license.

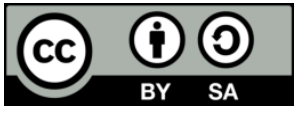

\section{Corresponding Author:}

Ismail Taha Ahmed

College of Computer Sciences and Information Technology

University of Anbar

Anbar, Iraq

Email: ismail.taha@uoanbar.edu.iq

\section{INTRODUCTION}

Various kinds of distortion such as noise, blurring, fast fading, blocking artifacts and contrast which may appear because of some of certain processes on the image can degrade the quality of images. Subjective and objective methods are two types of IQA or video quality assessment (VQA) used to evaluate the image quality $[1,2]$. In real-time applications is impractical to use subjective quality assessment because it takes time and is expensive. Therefore, objective IQA algorithms are the best solution since the role of human is limited in order to predict the quality of image. Objective IQAs can be grouped into full reference (FR), reduce reference (RR) and no reference (NR). These groups require the availability of original image [3-5].

Most of FR-IQA and RR-IQA applications are constrained due to available of original image. Therefore, NR-IQA is the best solution for this case. Recently, the existing NR-IQA works focusing on distorted images by compression, noise and blurring [6,7]. Contrast is one of the most popular forms of distortion. Figure 1 shows the contrast-distorted image is low gray scale image [8]. poor lighting condition and low quality image acquisition device can produce contrast distortion $[9,10]$. Unfortunately, the current work carried out in the area of NR-IQA for CDI is very minimal. 


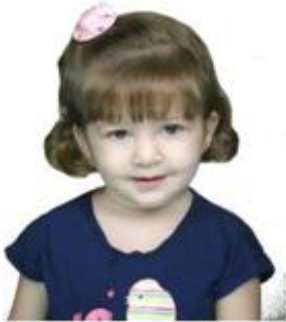

(a)

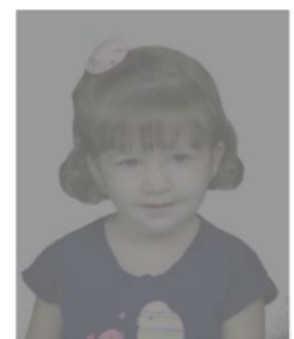

(b)

Figure 1. Contrast-distorted image, (a) good contrast image (my lena.bmp), and (b) poor contrast image [8]

Recently, the existing IQAs works for CDI are (1) RIQMC [11] based on entropies and image histogram order statistics; and (2) NR-IQA-CDI [12] which is constructed on the basis of NSS principles such that there are certain regularities in natural scene statistics that may be missing from the statistics of distorted images. Global spatial statistics features are used in NR-IQA-CDI including the mean, standard deviation, entropy, kurtosis and skewness. Regrettably, NR-IQA-CDI performance are not positive in some of test image databases, TID2013 and CSIQ, where the PLCC are only around 0.57 and 0.76, respectively.

The current NR-IQA-CDI is based on global spatial statistics which suffers from three problems. Secondly, spatial domain is lacking of info on scale and orientation which are essential for compact representation of curve which is a common structure in natural image. Compact representation is essential for analysis to be focused on relevant information to reduce confusion by irrelevant info or noise. It has been proven useful in image contrast enhancement and IQA.

Pyramid and wavelet transform is one of multiscale decomposition transform (MSD) has been used successfully in many image processing applications. However, WT lacks directionality. multiscale geometrical analysis (MGA) transforms [13] were proposed to resolve this issue. Curvelet transform (CT) decomposes the original image into set of frequency coefficients of subbands with various scales, orientation and location [14]. In Coarse part, Low-frequency coefficients are distributed. In Fine part, high frequencies coefficients are distributed. And the middle layer are distributed to detail. Curvelet has a common characteristics that distinguishes it from the other types of Transforms are Higher directional sensitivity and lower redundancy [15].

The first NR-IQA [16] used features derived from curvelet transform. Liu et al. [17] and Shen et al. [18] introduced a new NR-IQA based on CT. The success of all of these works rely on the strength of curvelet, in which CT provides a rich source of orientation information on images. Such info could be used to detect the presence of distortion in the image. Liu et al. [17] present an effective NR-IQA using energy features derived from the curvelet domain. There features were found closely related through various types of distortion to the natural image quality. However, all of these works proposed specially for quality evaluation of distorted images due to compression, noise, and blurring. This study is motivated by the strength of curvelet transform in efficient representation of curve, a basic structure often seen in natural scene images. It begins with exploring the use of curvelet domain features in characterizing contrast-distorted images (CDI). Therefore, this paper enhances the existing NR-IQA-CDI using curvelet domain features.

\section{CURVELET TRANSFORM}

By applying an effective parabolic scaling law: width $\cong(\text { length })^{2}$, the CT can better represent edges and other singularities along curves [13]. Horizontal, vertical and diagonal are limited directional information only captured in Wavelet transform. However, curvelet transform decompose the image with geometric bases in multiple directions and positions [13, 15]. Since the curvelet coefficients are categorized based on orientation and scale, the curvelet edge is smoother than the Wavelet edge. Figure 2 shows the edge representation in curvelet and wavelet transforms. It can be observe that curvelet edge can be represented by smaller amount of large coefficients, while wavelet edge can be represented by a bigger amount of large coefficients in the fine scale.

In last generation of curvelet transform, Cand'es et al. proposed a two fast discrete curvelet transforms (FDCT) i.e: Equation (1) unequally-spaced fast fourier transform (USFFT) based curvelet and (2) frequency wrapping based curvelet [15]. The DCT of a 2-D function $f[\mathrm{t} 1, \mathrm{t} 2]$ is formulated as:

$$
C^{D}(j, \ell, k):=\sum_{0 \leq t 1, t 2<n} f[t 1, t 2] \overline{\varphi_{J, \ell, k}^{D}[t 1, t 2]},
$$


where $\varphi, j, \ell, k$ are curvelet functions, scale, orientation and position respectively. $\mathrm{t} 1, \mathrm{t} 2$ denote coordinates in the spatial domain: $0 \leq \mathrm{t} 1, \mathrm{t} 2<\mathrm{n} . C^{D}(j, \ell, k)$ denotes curvelet coefficient. In our work, FDCT by wrapping is applied.
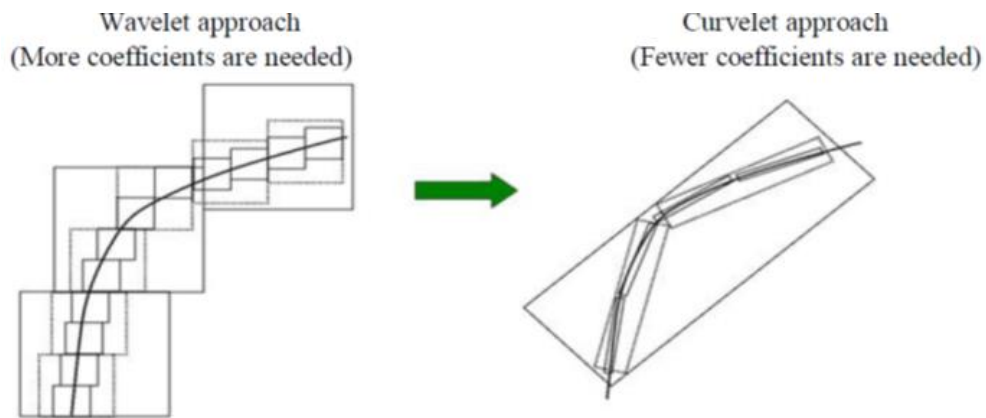

Figure 2. Wavelet and curvelet transforms edge representations [16]

\section{APPLICATION OF CURVELET DOMAIN FEATURES IN NR-IQA-CDI}

Here, we presents how curvelet domain features are applied in NR-IQA-CDI. It begins with trying to addressing the problems of current NR-IQA-CDI followed by formulating curvelet domain features and predicting image quality using the features.

\subsection{CDI distinguish}

$\mathrm{CT}$ is an effective image representation by smaller amount of large coefficients as compared to those in the spatial domain as mention in [19]. Large-magnitude coefficients only occur in regions of the image that contain fine details; fine scales coefficients show the existence local info in an image. But in the coarsest scale consists of mainly large-magnitude coefficients and matches the image in low spatial resolution. Overall contrast and brightness of an image are depends on the distribution of the coefficients. In an image with good contrast must be included a powerful overall contrast, relatively bright appearance and fine details. In order to represent the characteristics, easily can be accomplished by using distribution of curvelet coefficients. In good overall contrast and bright appearance, a distribution of coefficients at coarsest scale with high dispersion and high central tendency. While in presence of fine details, a distribution of coefficients at finer scale with high central tendency. Briefly, from the observation of the distributions of curvelet coefficients at the coarsest and finest scales can be achieved by generate possible features in characterizing good contrast images and also CDI [19]. Therefore, in the next section, the details about formulating the features from the curvelet coefficients will be presented.

\subsection{Formulating curvelet domain features}

This section presents the two main processes in the proposed NR-IQA-CDI rely on curvelet domain features (NR-IQA-CDI-CvT). The first process is for computing curvelet features and the second one is for predicting image quality using the statistics as explained in the sub-sections.

\subsubsection{Computing curvelet features}

There are 3 steps in computing curvelet features as shown in Figure 3.

Step 1: Pre-processing

In this step, Colour images are converted to grayscale image using (2), where $I_{\text {gray }}, I_{\text {red }} I_{\text {green }}$ and $I_{\text {blue }}$ are the grayscale image, and red, green and blue channel of the original RGB image, respectively. MATLAB $^{\odot}$ function rgb2gray () is used to perform the conversion.

$$
I_{\text {gray }}=0.2989 I_{\text {red }}+0.5870 I_{\text {green }}+0.1140 I_{\text {blue }}
$$

In addition, resize step was used to normalize the size of the input image to have the size of minimum dimension fixed to 256 in order to avoid mismatch of resolution during comparison.

Step 2: Fast discrete curvelet transform

Using FDCT, The grayscale image is partitioned into groups of curvelet coefficients $C(j, l, k 1, k 2)$ as defined by (3). The curvelet transformation was done using the curvelet 2.1.2 toolbox, available from http://www.curvelet.org. 


$$
C^{D}\left(j, l, k_{1}, k_{2}\right)=\sum_{\substack{0 \leq m<M \\ 0 \leq n<N}} f[m, n] \varphi_{j, l, k 1, k 2}^{D}[m, n]
$$

where $\varphi_{j, l, k 1, k 2}^{D}$ is a curvelet coefficients at different scales, $j$, orientations, $l$ and location, $(k 1, k 2)[20]$. Step 3: Extract features from curvelet coefficients

First, the magnitude of coefficients at each scale $j,\left|\theta_{j}\right|$ were computed. Coefficients from same scale but different orientations were grouped together. Next, the mean, $\mu()$ and standard deviation, $\sigma()$ of the curvelet coefficients at the two coarsest scales $j=\{1,2\}$ were extracted as the four features, $f_{1}, f_{2}, f_{3}$ and $f_{4}$ as defined by (4)-(7).

$$
\begin{aligned}
& f_{1}=\mu\left(\left|\theta_{1}\right|\right) \\
& f_{2}=\mu\left(\left|\theta_{2}\right|\right) \\
& f_{3}=\sigma\left(\left|\theta_{1}\right|\right) \\
& f_{4}=\sigma\left(\left|\theta_{2}\right|\right)
\end{aligned}
$$

The last feature, $f_{5}$, is the mean, $\mu()$ of the highest $1 \%$ curvelet coefficients, $\theta_{j}^{\prime}$ at the finest scale, $j=5$ as defined by (8):

$$
f_{5}=\mu\left(\left|\theta^{\prime}{ }_{5}\right|\right)
$$

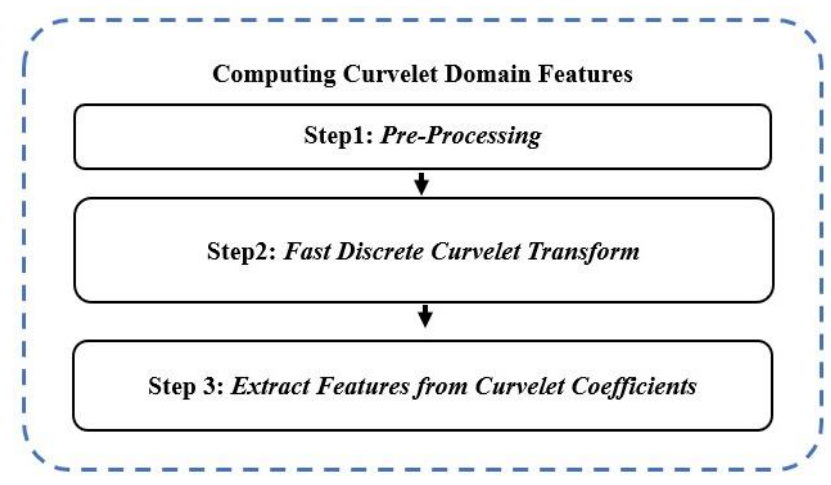

Figure 3. Flowchart of computing curvelet domain features

\subsubsection{Predicting image quality}

The steps for predicting image quality are shown in Figure 4.

Step 1 : Modelling using curvelet domain features

Step 1.1 : Compute curvelet domain features from training images

The step to compute curvelet domain features is as explained in section 3.2. The images used were from training set for modelling purposes.

Step 1.2 : Perform feature normalization and regression

In feature normalization, the values of each feature $f_{i}$ are normalized against their mean, $\mu_{f}$ and standard deviation, $\sigma_{f}$ such that the normalized values, $Z_{i}$ will have zero mean and unit standard deviation as defined in (9). Feature normalization is important before performing any machine learning such as regression because it tends to increase the accuracy.

$$
Z_{i}=\frac{f_{i}-\mu_{f}}{\sigma_{f}}
$$

The mean, $\mu_{f}$ and standard deviation, $\sigma_{f}$ and the regression function derived from this step will be used later in Step 2.2. Regression aims to find the mapping function, or better known as regression function, which map independent variables to dependent variables. In this work, the independent variables are the features and the dependent variable is the subjective mean opinion score (MOS). Regression is important in 
this work to remove non-linearity to improve the linear correlation between the features and MOS. In this work, support vector regression (SVR) (via LIBSVM-3.12 package [21]) is used to find the regression function, similar to what has been used in the current NR-IQA-CDI for fair comparison. In SVR, regression function is determined through the approach of supervised machine learning.

Step 2 : Computing image quality

Step 2.1 : Compute curvelet domain features from input image

The step to compute curvelet domain features is as explained in section 3.2. The image used is the input image which the quality is to be assessed.

Step 2.2: Compute image quality using the mean and standard deviation for feature normalization and regression function obtained from step 1.2

In this step, the curvelet domain features were normalized using the mean, $\mu_{f}$ and standard deviation, $\sigma_{f}$, obtained from Step 1.2. Next, they were plugged into the regression function obtained from Step 1.2 to compute the final image quality score.

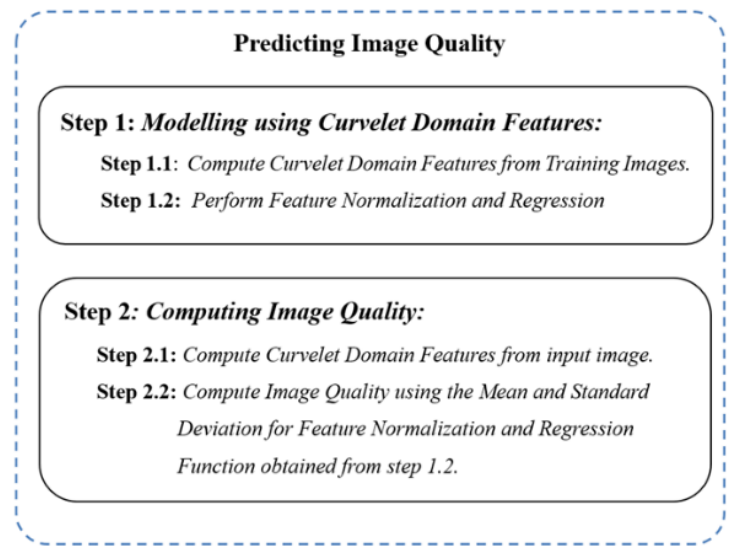

Figure 4. Flowchart of the predicting image quality

\section{EXPERIMENTAL RESULTS AND DISCUSSION}

Here, the NR-IQA-CDI rely on curvelet features (NR-IQA-CDI-CvT) performance is evaluated. The evaluation procedure, discussions, and conclusions was described.

\subsection{Evaluation procedure}

To evaluate our proposed, the same test image databases used by NR-IQA-CDI were used. Only contrast distorted images (116, 250, and 400) were chosen from CSIQ [22], TID2013 [23], and CID2013 [11], respectively. Mean opinion score (MOS) or differential mean opinion score (DMOS) are used to represent the Subjective scores. In order to evaluate the IQA accuracy, K-fold cross validation (CV) was used to determine how well the IQA could be generalized to independent data groups while decrease bias, since regression is essentially a learning algorithm that requires training.

Three databases were split randomly into 10 subgroups when performing the K-fold cross validation. To test the proposed metric, the method named 10-fold leave-one-out CV was used. By using $\mathrm{K}$ times, the result of the assessment were averaged. To decrease the variability, repeated rounds of cross-validation ( $\mathrm{k}=2$ to 10) were accomplished through various partitions $[8,24]$. The above cross-validation has been iterated hundred times (to prevent bias). Tables 1 and 2 show the average results. To evaluate IQA performance, three metric were used between the estimated objective scores and the subjective mean opinion scores (MOS). The metrics are (1) SROCC, (2) PLCC and (3) RMSE. The effective performance in case of correlation with human awareness if SROCC 1, PLCC 1 and RMSE 0 [8, 24].

\subsection{NR-IQA-CDI-CvT evaluation}

Tables 1 and 2 lists the three-performance metrics used to evaluate the current NR-IQA-CDI and the proposed NR-IQA-CDI-CvT with the three test image databases and $k$-fold cross-validation with $k$ range from 2 to 10. Figure 5 shows the bar chart graph comparing the average values of each of the threeperformance metrics of the two NR-IQA-CDI for each of the three databases. At a glance, NR-IQA-CDICvT outperformed the current NR-IQA-CDI in all the three-performance metrics using CSIQ and TID2013. 
Table 1. The PLCC, SROCC and RMSE across 100 train-test iteration via NR-IQA-CDI [12] (five global NSS features)

\begin{tabular}{llllllllll}
\hline $\mathrm{k}$ & & CSIQ & \multicolumn{3}{c}{ TID2013 } & \multicolumn{3}{c}{ CID2013 } \\
& PLCC & SROCC & RMSE & PLCC & SROCC & RMSE & PLCC & SROCC & RMSE \\
\hline 2 & 0.6849 & 0.7093 & 0.1233 & 0.5111 & 0.4450 & 0.8608 & 0.8685 & 0.8711 & 0.3090 \\
3 & 0.7518 & 0.7619 & 0.1121 & 0.5698 & 0.4886 & 0.8112 & 0.8844 & 0.8874 & 0.2945 \\
4 & 0.7389 & 0.7522 & 0.1133 & 0.5785 & 0.4992 & 0.8266 & 0.8799 & 0.8797 & 0.2970 \\
5 & 0.7923 & 0.7818 & 0.1092 & 0.5762 & 0.4957 & 0.8091 & 0.8738 & 0.8743 & 0.3035 \\
6 & 0.7601 & 0.7683 & 0.1097 & 0.5911 & 0.5173 & 0.7923 & 0.8804 & 0.8829 & 0.2979 \\
7 & 0.7840 & 0.7775 & 0.1045 & 0.5812 & 0.5024 & 0.8141 & 0.8857 & 0.8816 & 0.2917 \\
8 & 0.7724 & 0.7846 & 0.1070 & 0.5900 & 0.5028 & 0.8089 & 0.8818 & 0.8817 & 0.2961 \\
9 & 0.7788 & 0.7803 & 0.1054 & 0.5870 & 0.4932 & 0.8064 & 0.8844 & 0.8833 & 0.2962 \\
10 & 0.7978 & 0.7751 & 0.1076 & 0.5799 & 0.5048 & 0.8019 & 0.8789 & 0.8765 & 0.2974 \\
Av & 0.7623 & 0.7657 & 0.1102 & 0.5739 & 0.4943 & 0.8146 & 0.8797 & 0.8798 & 0.2981 \\
\hline
\end{tabular}

Table 2. The average PLCC, SROCC and RMSE across 100 train-test iteration via the NR-IQA-CDI-CvT (5 features)

\begin{tabular}{llllllllll}
\hline \multirow{2}{*}{$\mathrm{k}$} & & PLCC & SRIQ & & & TID2013 & & \multicolumn{3}{c}{ CID2013 } \\
& PROC & RMSE & PLCC & SROCC & RMSE & PLCC & SROCC & RMSE \\
\hline 2 & 0.8078 & 0.8076 & 0.1024 & 0.6681 & 0.5887 & 0.7360 & 0.8522 & 0.8566 & 0.3280 \\
3 & 0.8384 & 0.8354 & 0.0962 & 0.6875 & 0.6259 & 0.7144 & 0.8848 & 0.8882 & 0.2936 \\
4 & 0.8301 & 0.8358 & 0.0950 & 0.6881 & 0.6211 & 0.7213 & 0.8769 & 0.8823 & 0.3023 \\
5 & 0.8547 & 0.8417 & 0.0903 & 0.6882 & 0.6220 & 0.7242 & 0.8808 & 0.8866 & 0.2965 \\
6 & 0.8487 & 0.8396 & 0.0943 & 0.6955 & 0.6309 & 0.7095 & 0.8863 & 0.8896 & 0.2908 \\
7 & 0.8475 & 0.8351 & 0.0910 & 0.7044 & 0.6295 & 0.7073 & 0.8860 & 0.8880 & 0.2922 \\
8 & 0.8564 & 0.8473 & 0.0893 & 0.6914 & 0.6268 & 0.7177 & 0.8850 & 0.8890 & 0.2948 \\
9 & 0.8535 & 0.8396 & 0.0922 & 0.6991 & 0.6313 & 0.7024 & 0.8900 & 0.8926 & 0.2821 \\
10 & 0.8693 & 0.8536 & 0.0870 & 0.6928 & 0.6164 & 0.7197 & 0.8851 & 0.8954 & 0.2856 \\
Av & 0.8451 & 0.8373 & 0.0931 & 0.6906 & 0.6214 & 0.7169 & 0.8808 & 0.8854 & 0.2962 \\
\hline
\end{tabular}

Comparison Results on CSIQ Database

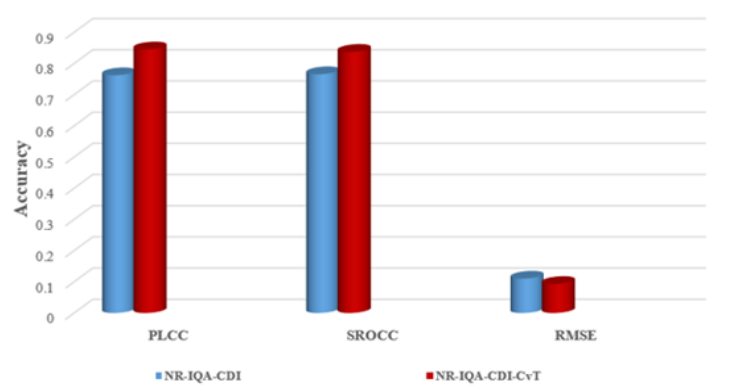

(a)

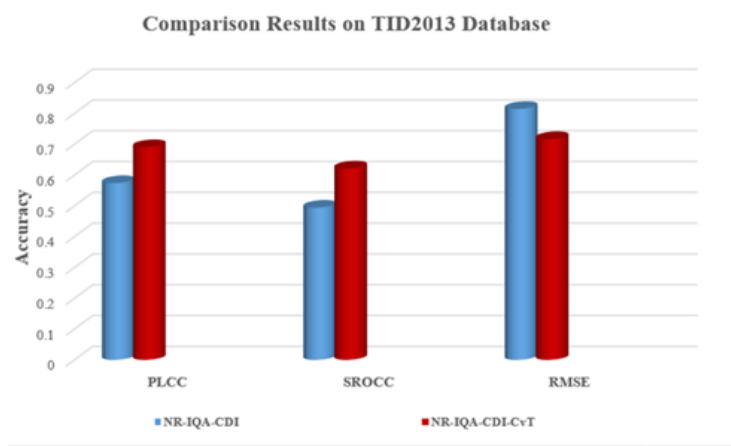

(b)

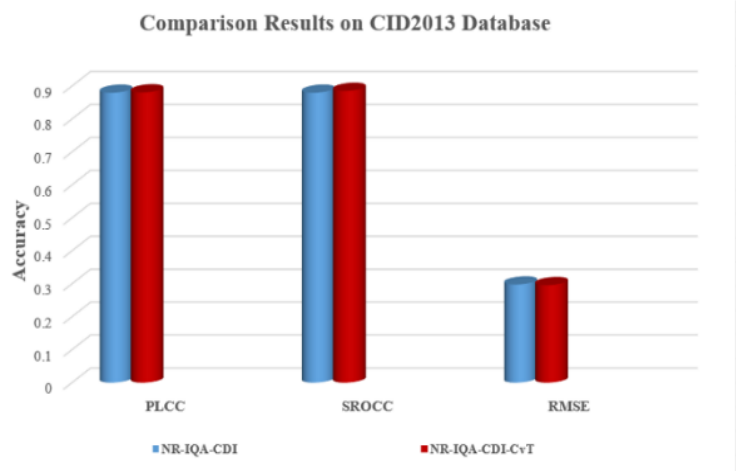

(c)

Figure 5. Comparison of SROCC, PLCC, and RMSE of NR-IQA-CDI, NR-IQA-CDI-CvT on; (a) CSIQ database, (b) TID2013 database, (c) CID2013 database 


\subsubsection{Statistical performance analysis}

\section{a. Percentage of difference}

by $(10)$ :

Each $k$ in each of the databases, the difference between the two-performance metrics are calculated

$$
d_{i}=\operatorname{cvtc}_{i}-c_{i}
$$

Where $c i$ corresponds to the first metric values without using features in curvelet domain and $c v t c i$ corresponds to the second metric values by using features in curvelet domain. Then the average percentage of differences is calculated for all the $\mathrm{k}$ values and databases. The percentage is calculated by dividing the performance difference by the absolute first metric value of ci.

$$
d p=\frac{1}{n}\left(\sum_{i=1}^{n} d_{i} / a b s\left(c_{i}\right)\right)
$$

Where $n$ corresponds to the total number $\mathrm{k}$ across all databases. The absolute value is used to keep the percentage (increment or decrement) sign of difference in performance. The percentage of difference is shown in Table 3.

Table 3. Percentage difference results for NR-IQA-CDI-CvT-NR-IQA-CDI

\begin{tabular}{cccc}
\hline Image Database & PLCC & SROCC & RMSE \\
\hline TID2013 & $20.49 \%$ & $25.81 \%$ & $-11.96 \%$ \\
CID2013 & $0.11 \%$ & $0.63 \%$ & $-0.67 \%$ \\
CSIQ & $10.99 \%$ & $9.41 \%$ & $-15.53 \%$ \\
All Databases & $10.53 \%$ & $11.95 \%$ & $-9.39 \%$ \\
\hline
\end{tabular}

\section{b. Statistical significance}

A paired T-test hypothesis test $[25,26]$ is implemented to the performance metric value calculated before and after adding curvelet features to produce the p-value as shown in Table 4. Generally, p-value of less than 0.05 implies that a significant difference appear within the values. Tables 4 and 5 displays the $\mathrm{P}$-values of the paired T-tests for adding curvelet feature.

Table 4. P-values of differences between NR-IQA-CDI \& NR-IQA-CDI-CvT

\begin{tabular}{cccc}
\hline Image Database & $P L C C$ & SROCC & $R M S E$ \\
\hline TID2013 & $1.24 \times 10^{-08}$ & $2.54 \times 10^{-10}$ & $1.48 \times 10^{-08}$ \\
CID2013 & $3.41 \times 10^{-01}$ & $5.43 \times 10^{-02}$ & $2.88 \times 10^{-01}$ \\
CSIQ & $4.34 \times 10^{-07}$ & $1.25 \times 10^{-07}$ & $4.32 \times 10^{-08}$ \\
All Databases & $1.85 \times 10^{-07}$ & $1.49 \times 10^{-07}$ & $4.73 \times 10^{-05}$ \\
\hline
\end{tabular}

Table 5. P-values of differences between NR-IQA-CDI \& NR-IQA-CDI-CvT If $p$-value $\leq 0.05$ : the observed difference is "significant"

\begin{tabular}{cccc}
\hline Image Database & PLCC & SROCC & RMSE \\
\hline TID2013 & Significant & Significant & Significant \\
CID2013 & Insignificant & Insignificant & Insignificant \\
CSIQ & Significant & Significant & Significant \\
All Databases & Significant & Significant & Significant \\
\hline
\end{tabular}

\subsubsection{Discussion}

The discussion on the results in Tables 3,4 , and 5 are as:

- Table 3 indicates that the findings using the TID2013 have improved, that was our main aim for enhancement. There was a significant increase in PLCC and SROCC by $20.49 \%$ and $25.81 \%$, respectively. The RMSE decreased noticeably by $11.96 \%$. The 3 p-values for TID2013 were below than 0.05 , meaning significant differences in those three performance measures as shown in Tables 4 and 5.

- For CID2013, PLCC and SROCC increased very marginally by $0.11 \%$ and $0.63 \%$, respectively. The RMSE also decreased very marginally by $0.67 \%$. The $3 p$-values for CID2013 indicate that the differences in these three measures were not statistically significant as shown in Tables 4 and 5. 
- $\quad$ For the CSIQ, there was a moderate increase in PLCC and SROCC by $10.99 \%$ and $9.41 \%$, respectively. The RMSE decreased moderately by $15.53 \%$. The $3 p$-values for CSIQ below than 0.05 , indicate that the differences in these three measures were statistically significant as shown in Tables 4 and 5.

For the average results of the three databases, there were moderate increase in PLCC and SROCC by $10.53 \%$ and $11.95 \%$, respectively. The RMSE decreased moderately by $9.39 \%$. The three $p$-values for all databases below than 0.05 , indicate that the differences in these three performance matrices were statistically significant as shown in Tables 4 and 5.

\section{CONCLUSION}

In this paper, the existing NR-IQA-CDI was enhanced using curvelet domain features. In characterizing a good contrast image and contrast-distorted image at various scales, distributions of curvelet coefficients were found to be accurate. The five curvelet domain features proposed were derived from the distribution of the curvelet coefficients across the two coarsest scales and the finest scale. The performance evaluation indicated that NR-IQA-CDI-CvT significantly outperformed the existing NR-IQA-CDI in database TID2013 and CSIQ, which were the primary target for improvement of this work, although there wasn't much performance difference in database CID2013.

\section{ACKNOWLEDGEMENTS}

This research is supported by UNITEN Research Fund for Journal Publication 2020.

\section{REFERENCES}

[1] N. Thakur and S. Devi, "A new method for color image quality assessment," International Journal of Computer Applications, vol. 15, no. 2, pp. 10-17, 2011.

[2] I. T. Ahmed C. S. Der, and B. T. Hammad, "A Survey of Recent Approaches on No-Reference Image Quality Assessment with Multiscale Geometric Analysis Transforms," International Journal of Scientific \& Engineering Research, vol. 7, no. 12, pp. 1146-1156, 2016.

[3] S. G. G. Mrak and others, "Reliability of objective picture quality measures," Journal of Electrical Engineering, vol. 55, no. 1-2, pp. 3-10, 2004.

[4] I. T. Ahmed, C. S. Der and B. T. Hammad, "Recent approaches on no-reference image quality assessment for contrast distortion images with multiscale geometric analysis transforms: a survey," Journal of Theoretical and Applied Information Technology, vol. 95, no. 3, pp. 561-569, 2017.

[5] S. TM and K. B. Ramesh, "An efficient computational approach to balance the trade-off between image forensics and perceptual image quality," International Journal of Electrical and Computer Engineering (IJECE), vol. 9, no. 5, pp. 3474-3479, 2019.

[6] T. M. Kusuma, R. Rahmanto and E. Haryatmi, "Adaptive power link adaptation on DVB-T system based on picture quality feedback," International Journal of Electrical and Computer Engineering (IJECE), vol. 9, no. 4, pp. 3121-3129, 2019.

[7] B. Bhatkalkar, A. Joshi, S. Prabhu, and S. Bhandary, "Automated fundus image quality assessment and segmentation of optic disc using convolutional neural networks," International Journal of Electrical and Computer Engineering (IJECE), vol. 10, no. 1, pp. 816-827, 2020.

[8] I. T. Ahmed, C. S. Der, N. Jamil and M. A. Mohamed, "Improve of contrast-distorted image quality assessment based on convolutional neural networks," International Journal of Electrical and Computer Engineering (IJECE), vol. 9, no. 6, pp. 5604-5614, 2019.

[9] R. C. Gonzalez and R. E. Woods, "Digital image processing," Upper Saddle River, NJ: Prentice Hall, 2012.

[10] T. Arici, S. Dikbas and Y. Altunbasak, "A histogram modification framework and its application for image contrast enhancement," IEEE Transactions on Image Processing, vol. 18, no. 9, pp. 1921-1935, 2009.

[11] K. Gu, G. Zhai, X. Yang, W. Zhang and M. Liu, "Subjective and objective quality assessment for images with contrast change," 2013 IEEE International Conference on Image Processing, Melbourne, VIC, 2013, pp. 383-387.

[12] Y. Fang, K. Ma, Z. Wang, W. Lin, Z. Fang and G. Zhai, "No-reference quality assessment of contrast-distorted images based on natural scene statistics," IEEE Signal Processing Letters, vol. 22, no. 7, pp. 838-842, 2015

[13] H. Führ, L. Demaret and F. Friedrich, "Beyond wavelets: New image representation paradigms," Doc. image compression, vol. 7, pp. 179-206, 2006

[14] E. J. Candès and D. L. Donoho, "New tight frames of curvelets and optimal representations of objects with piecewise C2 singularities," Communications on Pure and Applied Mathematics, vol. 57, no. 2, pp. 219-266, 2004.

[15] E. Candes, L. Demanet, D. Donoho and L. Ying, "Fast discrete curvelet transforms," SIAM Journal on Multiscale Modeling and Simulation, vol. 5, no. 3, pp. 861-899, 2006.

[16] J. Shen, Q. Li and G. Erlebacher, "Curvelet based no-reference objective image Quality Assessment," 2009 Picture Coding Symposium, Chicago, IL, 2009, pp. 1-4.

[17] L. Liu, H. Dong, H. Huang and A. C. Bovik, "No-reference image quality assessment in curvelet domain," Signal Processing: Image Communication, vol. 29, no. 4, pp. 494-505, 2014. 
[18] J. Shen, Q. Li, and G. Erlebacher, "Hybrid no-reference natural image quality assessment of noisy, blurry, JPEG2000, and JPEG images," IEEE Transactions on Image Processing, vol. 20, no. 8, pp. 2089-2098, 2011.

[19] I. T. A. Ahmed, C. S. Der Chen and B. T. H. Hammad, "Impact of Contrast-Distorted Image on Curvelet Coefficients," 2018 1st Annual International Conference on Information and Sciences (AiCIS), Fallujah, Iraq, 2018, pp. 28-32.

[20] T. Guha and Q. M. J. Wu, "Curvelet based feature extraction," in Face Recognition, InTech, 2010.

[21] C. Chang and C. Lin, "LIBSVM: a Library for Support Vector Machines (Version 2.3)," LIBSVM, pp. 1-39, 2001.

[22] E. C. Larson and D. M. Chandler, "Most apparent distortion: full-reference image quality assessment and the role of strategy," Journal of Electronic Imaging, vol. 19, no. 1, 2010.

[23] N. Ponomarenko, et al., "Color image database TID2013: Peculiarities and preliminary results," European Workshop on Visual Information Processing (EUVIP), Paris, 2013, pp. 106-111.

[24] I. T. Ahmed, C. S. Der, N. Jamil, and B. T. Hammad, "Analysis of Global Spatial Statistics Features in Existing Contrast Image Quality Assessment Algorithm," 2019 7th International Conference on Information and Communication Technology (ICoICT), Kuala Lumpur, Malaysia, 2019, pp. 1-4.

[25] D. J. Sheskin, "Handbook of parametric and nonparametric statistical procedures," Chapman \& Hall/CRC, p. 1776, 2003.

[26] Y. Fu and S. Wang, "A no reference image quality assessment metric based on visual perception," Algorithms, vol. 9, no. 4, pp. 87-108, 2016. 